\title{
DESIGN AND ANALYSIS OF A CAR BODY
}

\author{
Thiagarajan ${ }^{1}$, Sidhaant Rishi ${ }^{2}$, Sanskar Amit $^{3}$, Manish K Vedi ${ }^{4}$ \\ ${ }^{I}$ Assistant Professor, Automobile Engineering Department, SRM University Delhi-NCR campus, U.P, India \\ ${ }^{2}$ Student, Automobile Engineering Department, SRM University, Delhi-NCR campus, U.P, India \\ ${ }^{3}$ Student, Automobile Engineering Department, SRM University, Delhi-NCR campus, U.P, India \\ ${ }^{4}$ Student, Automobile Engineering Department, SRM University, Delhi-NCR campus, U.P, India
}

\begin{abstract}
The paper presents the method to design and analyze the concept car using modern software tools of CAD design and Analysis such as CFD, Draft and Zebra to get a much realistic model, which can be further used. The work is based on the elements of designing, aerodynamics, vehicle dynamic and other external influences on it. Also, model verification with numerical calculations was done to support the results. This paper presents the procedural and analytical methods to present and validate a car for its designing and aerodynamics.
\end{abstract}

Keywords: Aerodynamics, CFD, Designing, Autodesk CFD

\section{INTRODUCTION}

The times are changing so are the designs of the concept and the technology being installed in the cars. In this generation of high-speed automobile better aerodynamics play a major role in vehicle performance. This generates the demand of designing a vehicle with a new designing method supported by the fundamental procedures having the below mentioned qualities:-

- Simple

- Better Streamlining

- Higher Performance

- Good styling of the car body

In order to satisfy all the above needs certain design and engineering methods were implemented so that the model could achieve all the above mentioned aspects.

\subsection{Design}

1. For the design to be unique and artistic the unibody design was chosen as the main aspect of exterior designing. In the unibody design there are no panels and the exterior of the car is manufactured as one piece except the parts like the hood, trunk, roof and both the doors.

2. For a better proportions of the car, it was designed in the golden ratio or the divine ration of 1:1.618 in which the length : breadth and length: height ratio were having the same values. Due to this the proportions were set in a more meticulous fashion.

\subsection{Performance}

1. To produce a design with a cutting edge performance the most important part is to reduce drag and increase downforce. This was achieved by using a single stroke to design the side view curvature of the vehicle. This ensured that the car must obtain higher aerodynamic characteristics with the ease in design aspects.

2. To generate higher downforce the airflow was concentrated at the rear end of the car model.

\section{METHODOLOGY}

The Following shows the procedures to develop the CAD Design:

\subsection{Selection}

First is the selection of the type of car the latter wants to design such as a hatchback, a sports car, sedan or SUV etc. This paper represents the design of a sports car as the main focus is on the aerodynamic elements of a car to produce more power. Hence the design should be having lower ground clearance to receive a better traction and a better down force than a usual car. The ride height should be low to reduce the drag.

\subsection{Sketching}

After deciding the car type and general factors such as ground clearance, ride height sketching is done in which different views of the car are designed on to the paper according to the specification decided. It is one of the most crucial part it decides the major styling and designing elements of a car.

\subsection{CAD Model}

After the sketching part comes the main part of a design that is the CAD modeling[1] of the car, which is crucial for analysis, and giving the car a realistic look. Autodesk Fusion 360 Software was used, as it is very much known in industries for its high quality rendering graphics. This helps 
to give the car a more realistic outlook while presenting the car. For 3D modeling of the car Projections of the design canvases have to be placed. These projections can be the sketches made in different angles. They are placed in different planes according to their projections and the curvatures and lines are designed on those projections.

After the projections are drawn then they are turned into solid form using the sculpt environment. The sculptenvironment is utilized to form intricate shapes and curvatures easily. The shapes so formed in the sculpt environment gives a more realistic and better outlook to form better surfacing. The surface so formed can be a normal sheet body or a closed body. For the analysis to work only the closed body is required because CFD analysis only works when there is some thickness to the body otherwise the software package will assume the body to be open body and analysis will not be performed.

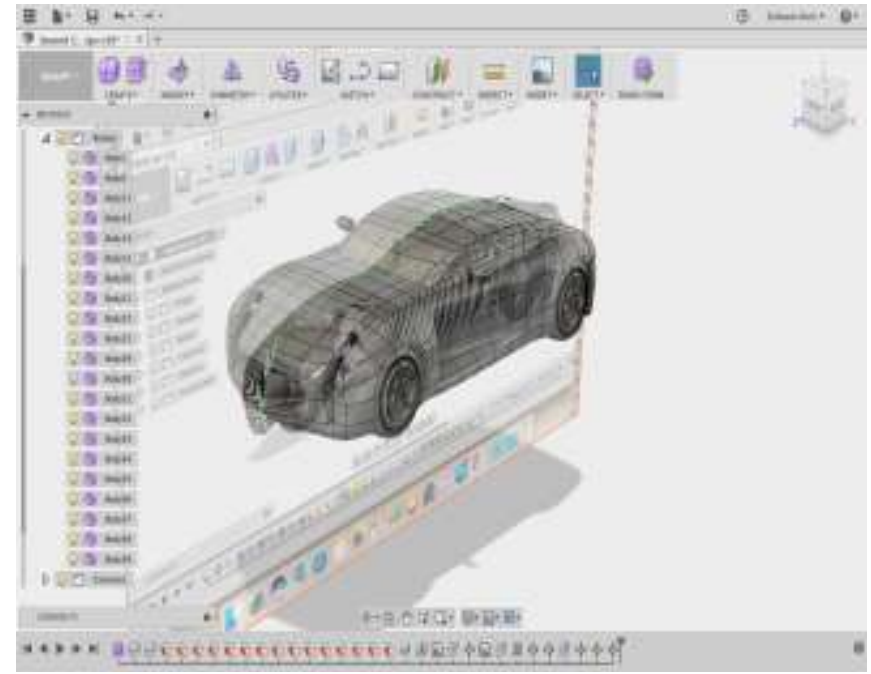

Fig- 1: Canvas placement

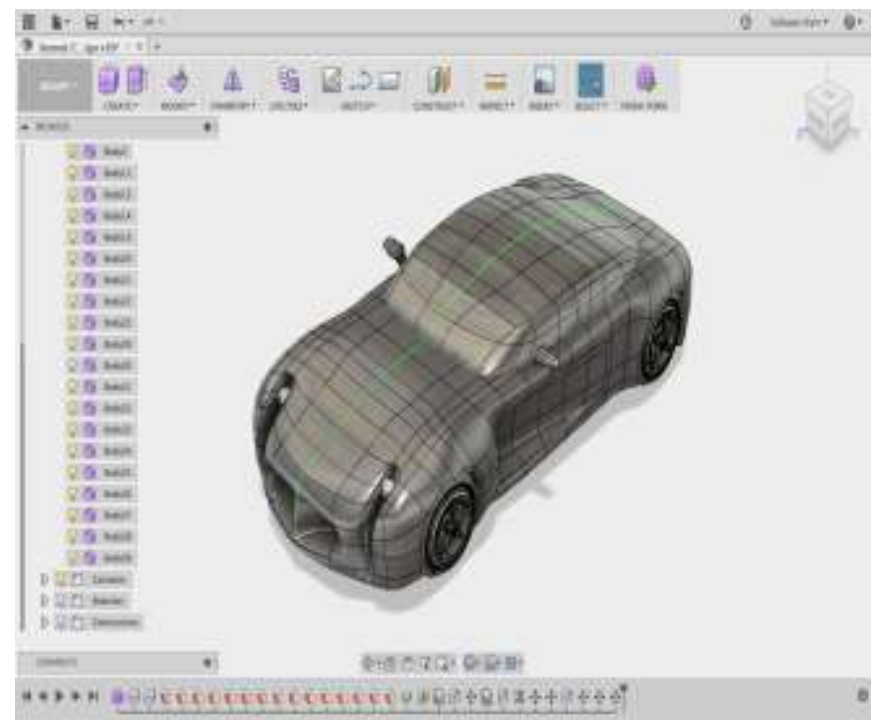

Fig- 2: CAD Model

\subsection{Curvature Analysis}

The Curvature analysis consists of analysis tools used to gain information on the type and amount of curvature on a surface. The Tools, which were used on this CAD model, were the zebra analysis and the Gaussian analysis.

\section{Zebra Analysis}

The Zebra analysis is the analysis tool used to check the continuity of the surfaces by applying the white and black stripes on the surfaces.

Interpreting the zebra analysis

G0 Position: This position of the surface edge is collocated, they touch but the tangency and curve of the surfaces do not match. Hence the zebra stripes do not match and results in a sharp edge curve.

G1 Tangency: The position and tangency of surfaces is the same. The zebra stripes line up but they move away when approaches towards the sharp curves.

\section{$\mathrm{G} 1(\mathrm{G} 0+\mathrm{G} 1$ or position + tangency)}

G2 Curvature: The position tangency and curvature of the surface edge is the same. The stripes line up and do not move away when approaching the sharp curves. The difference is very subtle and difficult to analyze.

\section{$\mathrm{G} 2(\mathrm{G} 0+\mathrm{G} 1+\mathrm{G} 2$ or position+ tangency+ curvature)}

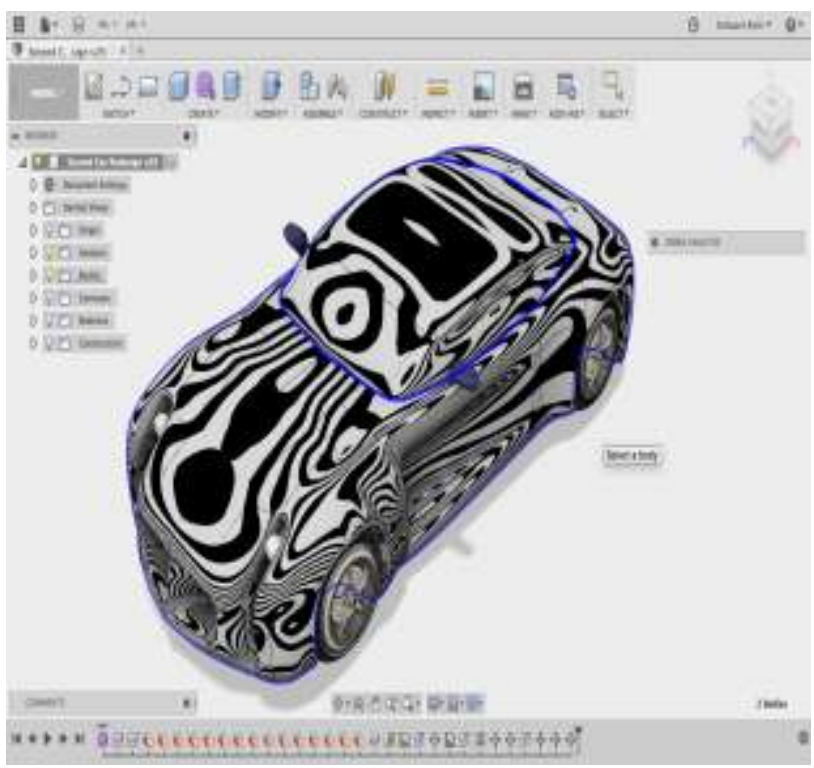

Fig- 3: Isometric View of CAD Model 


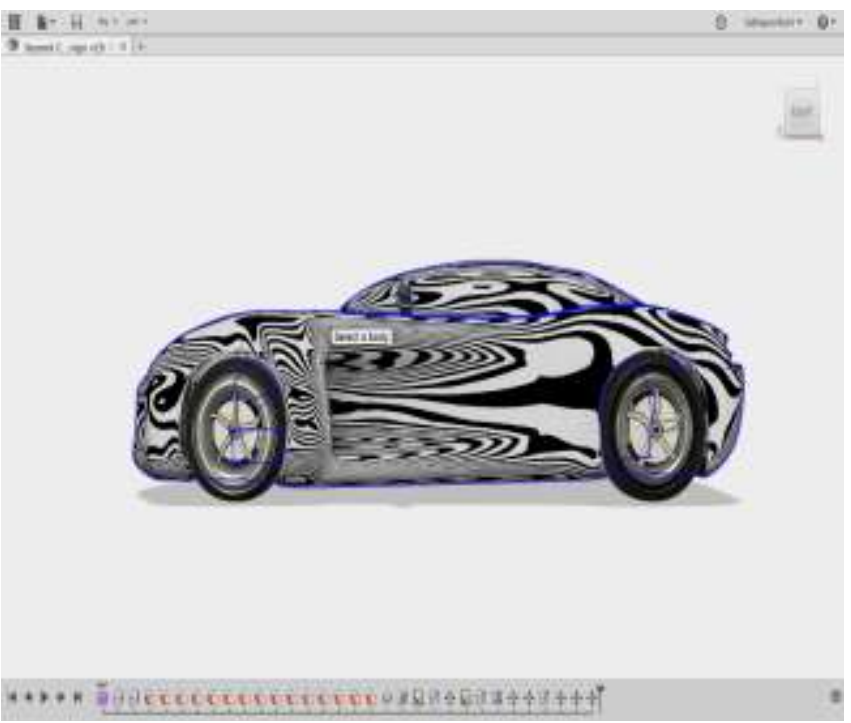

Fig- 4: Side View of CAD Model

The CAD Model proved to be symmetric and continuous also the zebra analysis showed that the surfaces generated was smooth so that less drag will be generated throughout the curvature of the car.

Gaussian Analysis: In differential geometry the Gaussian curvature is the product of the principal curvatures $\kappa_{1}$ and $\kappa_{2}$.

$$
K=\kappa_{1} \kappa_{2}
$$

There are three cases to interpret a Gaussian curvature:

1. Zero Curvature: The Gaussian curvature will be zero for a flat surface and it will be represented by green color on the vehicle.

2. Positive curvature: The Positive curvature will be obtained when the product of the principal curvature is positive as in the case of a sphere where the Gaussian curvature so obtained is equal to $1 / r^{2}$

3. Negative Curvature: The Negative Gaussian curvature will be obtained when the principal curvature are of opposite signs and has a negative curvature as in the case of a hyperboloid or inside surface of a torus.

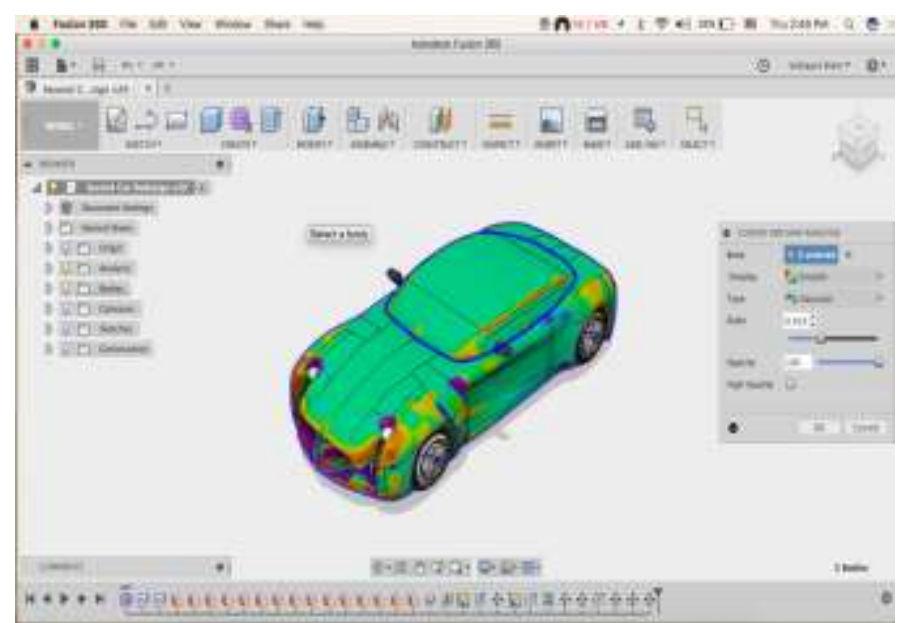

Fig- 5: Isometric View of CAD Model

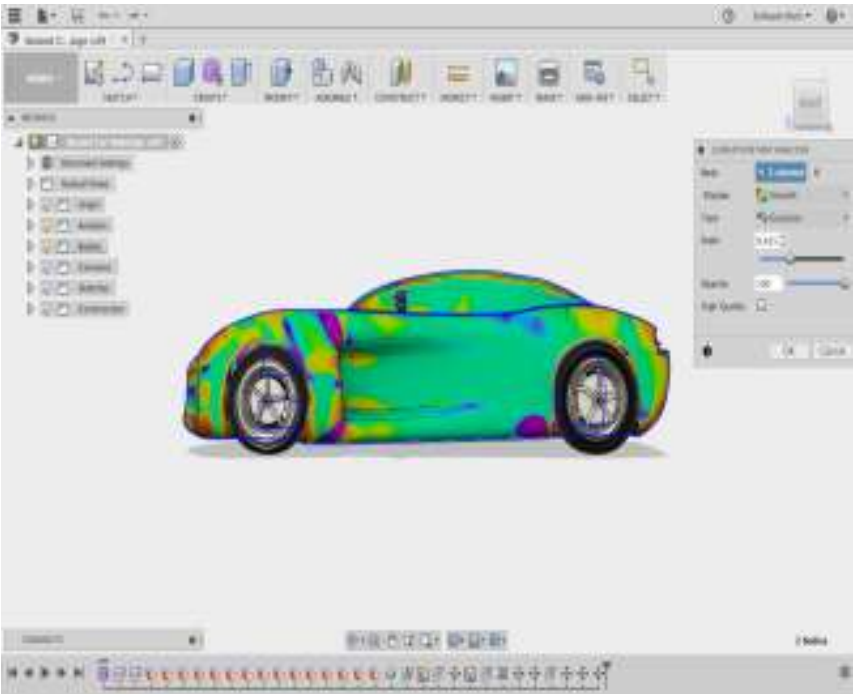

Fig- 6: Side View of CAD Model

Maximum surfaces of the car were flat hence zero curvature with some parts such as the curvature near side vent and near headlights were having negative curvature as due to their intricate geometry the design possessed a higher negative curvature. Overall the car proved to be flat and overall Gaussian curvature to be zero.

\section{COMPUTATIONAL FLUID DYNAMICS}

Computational fluid dynamics is a necessary component to study the aerodynamic component of the vehicle. It helps to study the airflow over the car body, The pressure distribution over the surface of the car and also to calculate the results of the vehicle such as drag, lift and lateral forces acting on the car.

The Computational dynamics include the general flow equations[2] :

\section{Continuity Equation}

$$
\frac{D \rho}{D_{t}}+\rho \frac{\partial U_{i}}{\partial x_{i}}
$$

\section{Momentum Equation}

$$
{\underset{\mathrm{I}}{\mathrm{I}}}_{\rho}^{\frac{\partial U_{j}}{\partial t}}+\rho U_{i} \frac{\partial U_{j}}{\partial x_{i}}=-\frac{\partial P}{\partial x_{j}}-\frac{\partial \tau_{i j}}{\partial x_{i}}+\rho g_{j}
$$

Where

$$
\tau_{i j}=-\mu\left[\frac{\partial U_{j}}{\partial x_{i}}+\frac{\partial U_{i}}{\partial x_{j}}\right]+\frac{2}{3} \delta_{i j} \mu \frac{\partial U_{k}}{\partial x_{k}}
$$

I: Local Change with time

II: Momentum Convection

III: Surface Force 
IV: Molecular dependent momentum Exchange (Diffusion) V: Mass Force

\section{Energy Equation}

$$
\rho c_{\mu} \frac{\partial T}{\partial t}+\rho c_{\mu} U_{i} \frac{\partial T}{\partial x_{i}}=-P \frac{\partial U_{i}}{\partial x_{i}}+\lambda \frac{\partial^{2} T}{\partial x_{i}^{2}}-\tau_{i j} \frac{\partial U_{j}}{\partial x_{i}}
$$

Where

I: Local Energy Change with time

II: Convection Term

III: Pressure work

IV: Heat Flux (Time)

$\mathrm{V}$ : Irreversible transfer of mechanical energy into heat

\section{Navier Stokes Equation}

We can combine all the above equation to form a general equation, which comprises of all the three equations

$$
\frac{\partial(\rho \phi)}{\partial t}+\frac{\partial}{\partial x_{i}}\left(\rho U_{i} \emptyset-\Gamma_{\varnothing} \frac{\partial \emptyset}{\partial x_{i}}\right)=q_{\varnothing}
$$

When $\varnothing=1, U_{j}, T$ we can respectively get continuity equation, momentum equation, energy equation.

These are the general equation, which helps to solve the CFD problems.

\subsection{Material}

The Material is applied to the car parts such as the car body such the car body is having carbon fiber material. Rubber is applied for tires etc.

\subsection{Boundary Layer Condition}

The Boundary layer Condition is applied to the wall around the model to simulate the actual conditions.

\subsection{Velocity}

The velocity was taken along the longitudinal direction flowing towards the car. In this case the different values are taken into consideration that is $60,120,160$ to obtain final and more precise results

\subsection{Slip Symmetry}

The slip symmetry was applied as the car is having the mirror symmetry along its longitudinal plane.

\subsection{Pressure}

The Pressure condition is applied at the rear end of the car with the value of $0 \mathrm{~Pa}$ in this case.

\subsection{Meshing}

The fine meshing was applied to obtain higher accuracy results. The total number of nodes so formed is 1540371 out of which 1378071 were fluid nodes and 162300 solid nodes. The total number of elements formed is 6535723 out of which 5133674 are fluid elements and 1402048 are solid elements. The model is having 1 Inlet and 1 outlet.

The model has undergone various meshing procedure that are as follows

\section{Surface Meshing}

First the surface meshing takes place as in the model an overall 969 surface meshes were formed. After the surface meshing is done surface smoothing takes place to generate a smooth mesh.

\section{Volume Meshing}

After the surface mesh takes place the CAD model undergoes the volume meshing in which the meshing occurs in a $3 \mathrm{D}$ geometry to mesh the depth of the CAD model.

\subsection{Solving}

After the meshing the solver undergoes the solution process in which the software package prepares the solution for the given $\mathrm{CAD}$ model using the above-mentioned general equations.

When the solution is created various analysis can be performed on the CAD model as in our example we did flow simulation using traces and Iso surfaces.

\subsection{Flow Simulation}

In the flow simulation we can see the path in which air flows by applying the trace sets to the wall. By applying the same we are representing the airflow, which will take place in the actual environment. This helps to study the behavior model while placing it in the conditions similar to the actual environment. In the model the flow simulation took place at three different air velocities i.e. of $60 \mathrm{kmph}, 120 \mathrm{kmph}$, $160 \mathrm{kmph}$. Three different speeds were used to get more precise results.

In the simulations two different studies were utilized which are mentioned below:-

Wind Tunnel Simulation: In the wind tunnel simulation grid of traces of size $(11 \times 6)$ was used. These trace points act as a source of wind flow lines. These lines help to judge the airflow across various parts of the model.

It helps to establish an idea of judgment for Turbulence across various parts of the model.

Pressure analysis: The static pressure across the vehicle can be depicted using two methods i.e. by adding planes or by adding iso surfaces. It helps to judge the pressure induced on the car while travelling at various speeds. Pressure induced should not be high at one end and low at the other,as it would create instability across the vehicle. 


\section{CFD POST PROCESSING AND RESULTS}

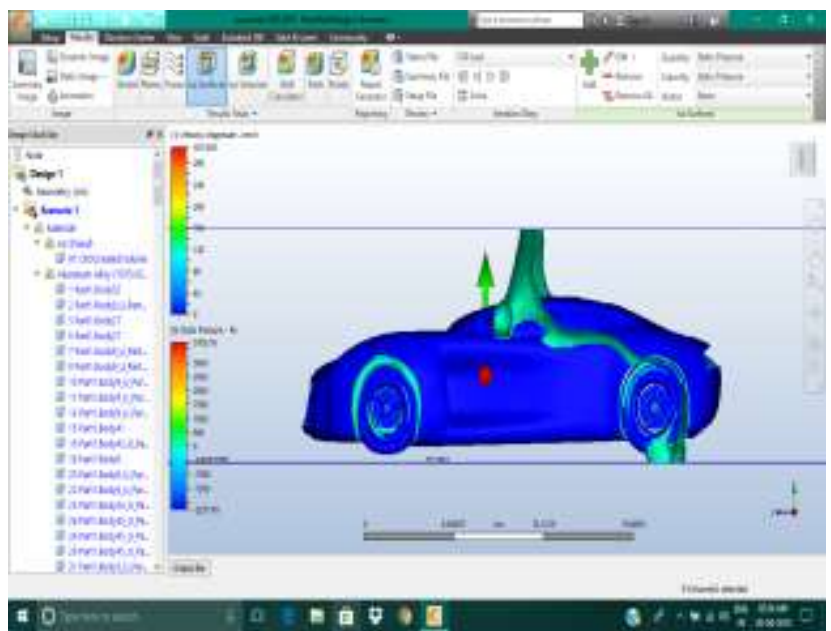

Fig- 7: Iso plane pressure analysis

This analysis uses the isometric plane to obtain different pressure values at various parts of the model. As in the above scenario the pressure is induced at the wheel arches and at the top of the car body which depicts the downforce as there is no pressure induced at the bottom of the vehicle depicting that lift force is very low.

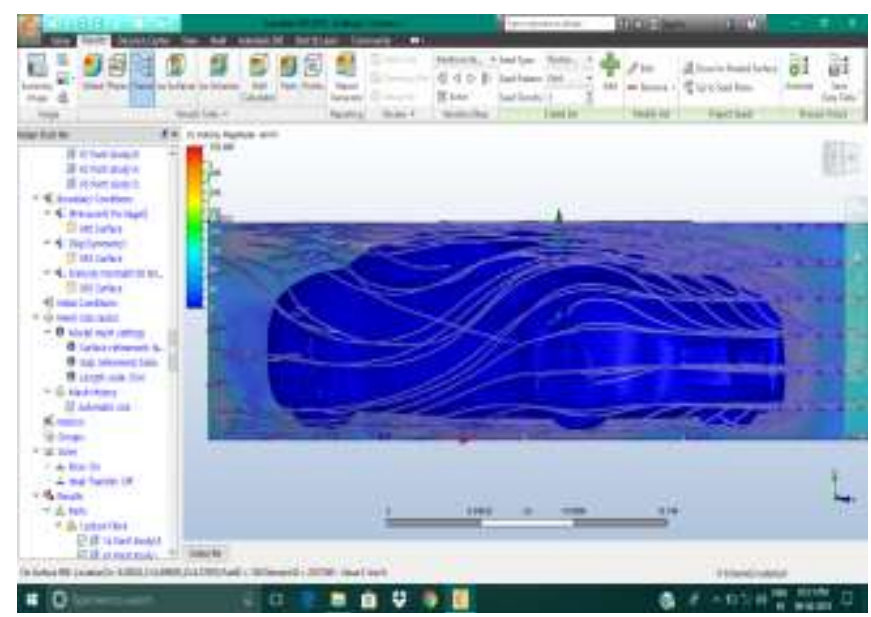

Fig- 9: Wind Tunnel Airflow Simulation

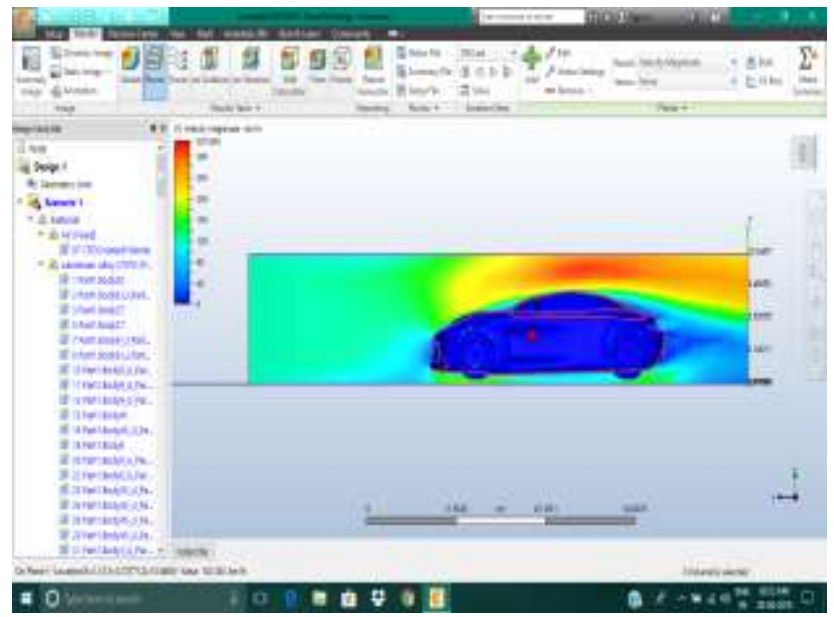

Fig- 8: Plane velocity analysis
The wind tunnel airflow and the plane view is obtained by adding traces and a plane respectively. Plane velocity analysis represents the velocity variation of different layers of air across the car body whereas the wind tunnel simulation represents the same besides representing the airflow pattern across the car.

Table 1: Results of the analysis

\begin{tabular}{|l|l|l|l|}
\hline $\begin{array}{l}\text { Air Velocity in } \\
\text { wind tunnel }\end{array}$ & $60 \mathrm{~km} / \mathrm{hr}$ & $120 \mathrm{~km} / \mathrm{hr}$ & $160 \mathrm{~km} / \mathrm{hr}$ \\
\hline $\begin{array}{l}\text { Frontal area of } \\
\text { our car model }(\mathrm{A})\end{array}$ & $2.08 \mathrm{~m}^{2}$ & $2.08 \mathrm{~m}^{2}$ & $2.08 \mathrm{~m}^{2}$ \\
\hline $\begin{array}{l}\text { Air Drag force } \\
\left(\mathrm{F}_{\mathrm{d}}\right)\end{array}$ & $142.8 \mathrm{~N}$ & $577.89 \mathrm{~N}$ & $1054 \mathrm{~N}$ \\
\hline Lift Force $\left(\mathrm{F}_{\mathrm{l}}\right)$ & $40.8 \mathrm{~N}$ & $285 \mathrm{~N}$ & $204 \mathrm{~N}$ \\
\hline $\begin{array}{l}\text { Drag coefficient } \\
\left(\mathrm{C}_{\mathrm{d}}\right)\end{array}$ & 0.406 & 0.41 & 0.41 \\
\hline $\begin{array}{l}\text { Lift Coefficient } \\
\left(\mathrm{C}_{\mathrm{l}}\right)\end{array}$ & 0.16 & 0.27 & 0.11 \\
\hline Downforce & $35.6 \mathrm{~kg}$ & $200 \mathrm{~kg}$ & $582.5 \mathrm{~kg}$ \\
\hline
\end{tabular}

The drag force increases with the speed of the air velocity whereas the lift force first increases then decreases due to the asymmetry of the car along its longitudinal when viewed from side view. The Positive concave shape results in the higher downforce values as the pressure above the car becomes higher than the pressure under the car both of which are generated by the air flowing across the car model. The graphs of different variables at different of the car are mentioned below: -

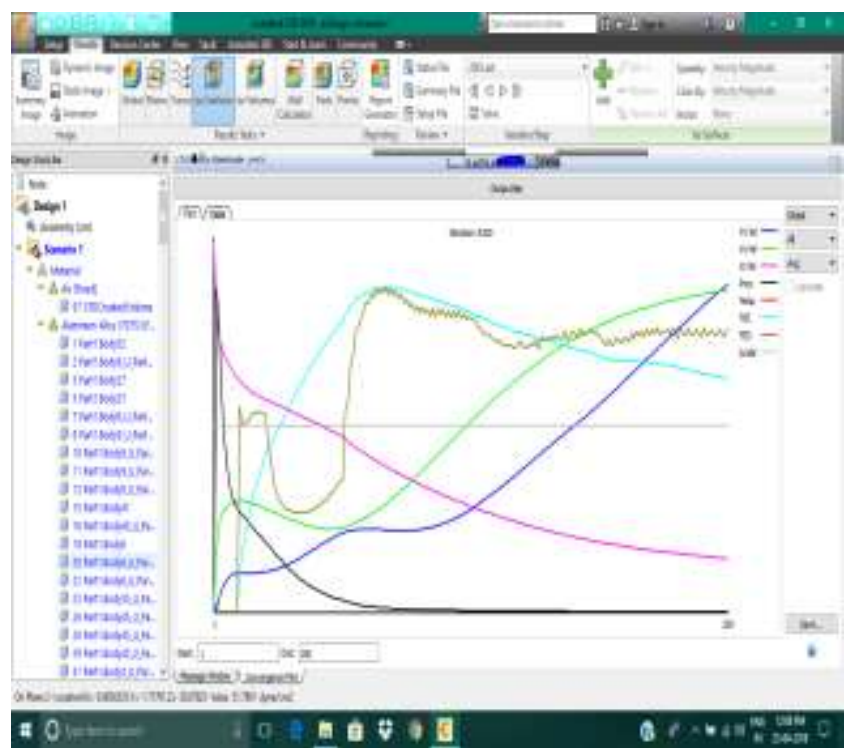

Fig- 9: At speed $60 \mathrm{~km} / \mathrm{hr}$ 


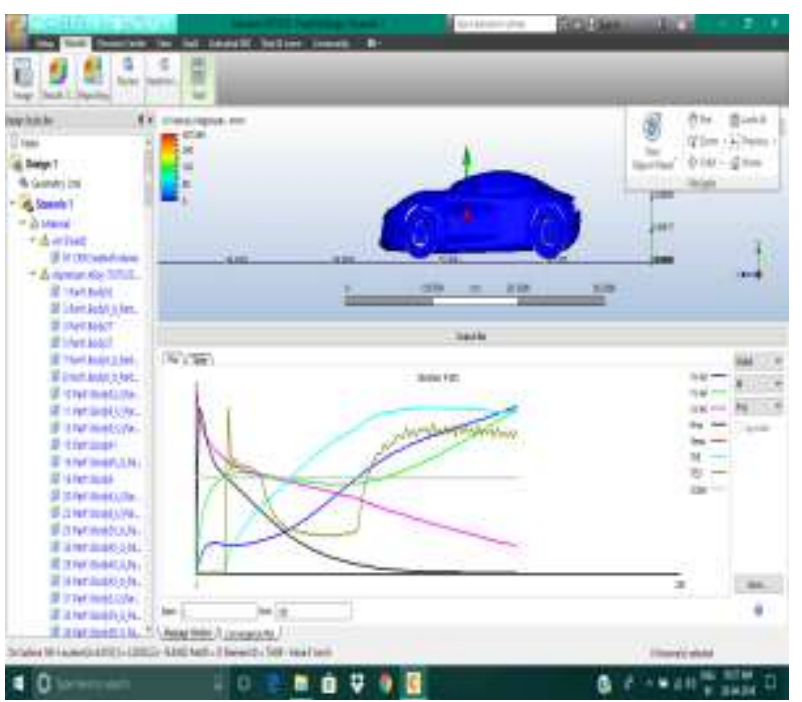

Fig- 10: At speed $120 \mathrm{~km} / \mathrm{hr}$

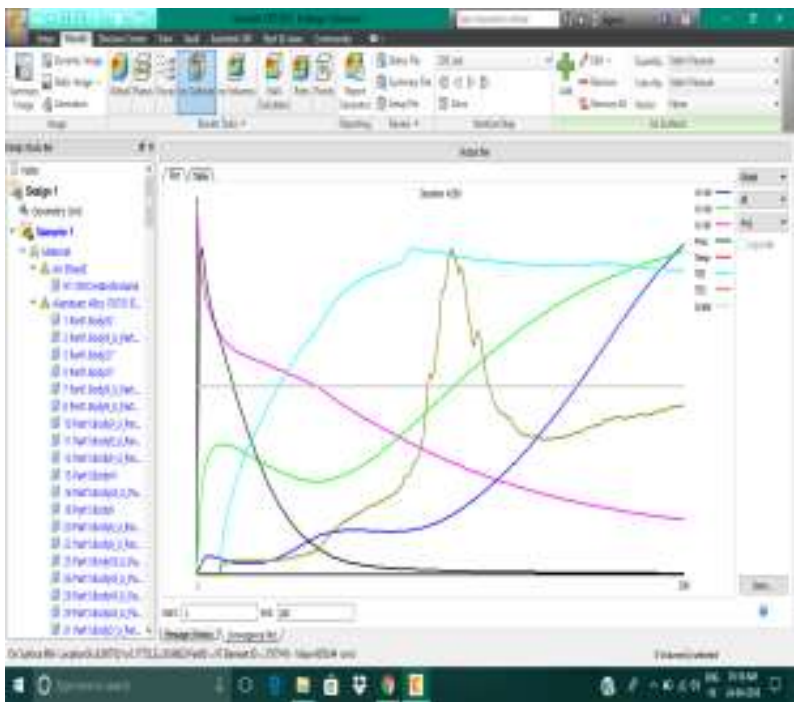

Fig- 11: At speed $160 \mathrm{~km} / \mathrm{hr}$

In the above-mentioned graphs there are eight variables, which include: -
1. $\mathrm{Vx}$
2. $\mathrm{Vy}$
3. $\mathrm{Vz}$
4. Pressure
5. Temperature
6. Total Kinetic Energy
7. Total Energy Dissipation
8. Scalar

The graphs resulted in a constant scalar quantity with reducing the pressure across the vehicle. The total energy dissipation is increased at the middle as the maximum velocity of air across its different layers is found in the region above the roof of the car. Total kinetic energy increases then tends to remain constant with slight deformations throughout the graph depicting a constant energy.
The Downforce increases as the speed increases and this helps in maintaining the stability of the vehicle at higher speeds. The downforce results in the higher cornering force as well as to maintain a better traction. The lift coefficient being reduced while the speed is increased helps to generate a higher downforce to maintain the traction and control over the car at higher speeds.

\section{CONCLUSION}

According to the data available from reading and calculations that the car model designed is a higher performance vehicle to due to its reduction in weight with the elegance of style making the car one of its kind. While comparing to other sports vehicle it generates a higher downforce value of $582.5 \mathrm{~kg}$, which keeps the car stable even when driving at high speeds. The car does not compromise with any of the performance and styling attributes. The design has unibody, which makes the car lighter and stronger as the unibody exterior will also help to take some load help enhancing the safety capabilities as well. This makes the car model to overall car with high performance, style and safety.

\section{REFERENCES}

[1] StjepanGalamboš, Jovan Dorić “ Design And Analysis of A Car Body Using CFD Software", Research Gate Publication, pp.- 693-696, Serbia, May 2015.

[2] DarkoDamjanović, DražanKozak, MarijaŽivić, ŽeljkoIvandić, TomislavBaškarić "CFD of a Concept Car in order to improve its Aerodynamics" Jármüipariinnováció Publication, pp.- 108-115, Croatia, Jan 2011. 